\title{
Alternative splicing after gene duplication drives CEACAM1-paralog diversification in the horse
}

Sophie Mißbach ${ }^{1,2}$, Denis Aleksic ${ }^{1}$, Lisa Blaschke ${ }^{1}$, Timm Hassemer ${ }^{1,3}$, Kyung Jin Lee ${ }^{1,4}$, Martin Mansfeld ${ }^{1}$, Jana Hänske', Johannes Handler ${ }^{5}$ and Robert Kammerer ${ }^{1,6^{*}}$

\begin{abstract}
Background: The CEA gene family is one of the most rapidly evolving gene families in the human genome. The founder gene of the family is thought to be an ancestor of the inhibitory immune checkpoint molecule CEACAM1. Comprehensive analyses of mammalian genomes showed that the CEA gene family is subject to tremendous gene family expansion and contraction events in different mammalian species. While in some species (e.g. rabbits) less than three CEACAM1 related genes exist, were in others (certain microbat species) up to 100 CEACAM1 paralogs identified. We have recently reported that the horse has also an extended CEA gene family. Since mechanisms of gene family expansion and diversification are not well understood we aimed to analyze the equine CEA gene family in detail.
\end{abstract}

Results: We found that the equine CEA gene family contains 17 functional CEACAM1-related genes. Nine of them were secreted molecules and eight CEACAMs contain transmembrane and cytoplasmic domain exons, the latter being in the focus of the present report. Only one (CEACAM41) gene has exons coding for activating signaling motifs all other CEACAM1 paralogs contain cytoplasmic exons similar to that of the inhibitory receptor CEACAM1. However, cloning of CDNAs showed that only one CEACAM1 paralog contain functional immunoreceptor tyrosinebased inhibitory motifs in its cytoplasmic tail. Three receptors have acquired a stop codon in the transmembrane domain and two have lost their inhibitory motifs due to alternative splicing events. In addition, alternative splicing eliminated the transmembrane exon sequence of the putative activating receptor, rendering it to a secreted molecule. Transfection of eukaryotic cells with FLAG-tagged alternatively spliced CEACAMs indicates that they can be expressed in vivo. Thus detection of CEACAM41 mRNA in activated PBMC suggests that CEACAM41 is secreted by lymphoid cells upon activation.

Conclusions: The results of our study demonstrate that alternative splicing after gene duplication is a potent mechanism to accelerate functional diversification of the equine CEA gene family members. This potent mechanism has created novel CEACAM receptors with unique signaling capacities and secreted CEACAMs which potentially enables equine lymphoid cells to control distantly located immune cells.

Keywords: Alternative splicing, Gene duplication, Horse, CEA gene family, CEACAM, Signaling motifs, Evolution

\footnotetext{
* Correspondence: Robert.Kammerer@fli.bund.de; Robert.Kammerer@fli.de

${ }^{1}$ Institute of Immunology, Friedrich-Loeffler-Institut, Suedufer 10, Greifswald, Insel Riems, Germany

${ }^{6}$ Friedrich-Loeffler-Institut, Bundesforschungsinstitut für Tiergesundheit,

Federal Research Institute for Animal Health, Südufer 10, D, 17493 Greifswald,

Insel Riems, Germany

Full list of author information is available at the end of the article
} 


\section{Background}

The Carcinoembryonic Antigen (CEA)-related cell adhesion molecule 1 (CEACAM1) is a multifunctional cell surface molecule of the immunoglobulin super family (ISF) involved in cell-cell adhesion, vascular remodeling, insulin resistance and immune responses. Two main splice forms of CEACAM1 concerning its cytoplasmic tail were identified. Isoforms with the long cytoplasmic tail provide inhibitory signals via immunoreceptor tyrosine-based inhibitory motifs (ITIM = S/I/V/LxYxxI/V/L) and immunoreceptor tyrosine-based switch motifs (ITSM $=$ TxYxxIV), while the short isoforms do not contain ITIMs/ITSM. Expression of the long isoform dominates in immune cells and the short isoform in epithelial cells, respectively $[1,2]$. Various pathogens recruit CEACAM1 as a cellular receptor to invade their hosts and at the same time modify the immune response [3-6]. There is growing evidence that an early hallmark of $C E A$ gene family expansion was the generation of paired receptors by creating a CEACAM1 paralog which has a very similar ligand-binding domain but transduce contrary, i.e. activating, signals into the cell as a countermeasure to pathogen attacks $[7,8]$. Once such a receptor pair was created, further expansion of the gene family is a critical process, since it may lead to an imbalance of paired receptor signaling. Most likely further diversification of the membrane anchorage and the signaling capacity was a prerequisite for further gene family expansion. Indeed, despite the sometimes tremendous expansion of the CEA gene families, the most populous families are found in certain bat species, containing up to 100 CEACAMs [9], the number of ITIM bearing CEACAMs in one species seem to be strictly limited. In most species only one CEACAM exist which contains ITIM in its cytoplasmic tail. Exceptions of this rule, described so far are mice and xenopus tropicalis, which have two CEACAMs with ITIM motifs http://www.carcinoembryonicantigen.de/mouse/index.html [8]. The two different ITIM bearing CEACAMs in mice, i.e. CEACAM1 and CEACAM2 have a different expression pattern, excluding a simple duplication of the inhibitory signals in a given cell type [10]. In addition further expansion of the CEA gene family in mice took place by duplication of CEACAMs lacking transmembrane and cytoplasmic domain exons. Although there is an amplification of the inhibitory receptor in humans, none of the paralogous genes encode a functional ITIM [11]. This was achieved by rendering the transmembrane domain of CEACAM1 into a signal peptide for a glycosylphosphatidylinositol (GPI) anchor. For this modification only minimal mutations are required including the introduction of a stop codon in the transmembrane domain exon [12]. No amplification of the inhibitory receptor takes place in the dog genome, but an amplification of activating CEACAMs was observed [13, 14]. Hence, efficient mechanisms must exist for the diversification of signaling capacities, if a duplication of inhibitory receptors is envisaged. We have recently reported that convergent evolution within the CEA gene families of humans and the horse had led to a similar expansion of secreted pregnancy-specific glycoproteins (PSG), which are a subgroup of CEACAM1 paralogous genes [15-17]. Obviously, secreted CEACAMs, which do not contain a transmembrane and cytoplasmic domain do not transmit signals into the cell and therefore are functionally different from the ancestral inhibitory receptor CEACAM1. Now we have analyzed the evolution of equine CEACAM genes containing a transmembrane domain exon and focused on mechanisms of their putative functional diversification. We observed that the expansion of the equine CEA gene family is due to the amplification of inhibitory CEACAM receptor genes. Characterization of the transcribed equine CEACAM1 paralog mRNAs revealed that only two CEACAMs have an ITIM motif and that alternative splicing (AS) after gene duplication (GD) is an important mechanism for functional diversification of duplicated membrane anchored CEACAMs in the horse.

\section{Results}

Comparison of equine CEACAM transmembrane domains

In the horse genome, CEACAM1 and seven CEACAM1 paralogs exist, which contain exons coding for transmembrane domains [15]. Phylogenetic analyses using nucleotide sequences of equine CEACAM transmembrane (TM) domain exons and of human CEACAM1 and $C E A C A M 3$, respectively, resulted in trees that comprise two deep clades, one containing human $C E A$ CAM1 TM sequence and 6 closely related equine TM sequences and the other containing human CEACAM3 TM sequence and a single equine TM sequence of $C E A$ CAM41 (Fig. 1a). Using the amino acid sequences for phylogenetic analyses, the CEACAM1 related TM could be separated into three subgroups (Fig. 1b). Subgroup 1 contains CEACAM1 and CEACAM43, the second subgroup consists of CEACAM42 and CEACAM50, and the third subgroup is composed of CEACAM45, CEACAM53 and CEACAM54. The latter three transmembrane domain exons are harboring a stop codon. For all predicted transmembrane domains complete transmembrane helices were predicted by the TMHMM Server, and arguing against the presence of GPI-anchored CEACAMs in the horse. Indeed, analysis of the sequences for the presence of GPI anchors using the PredGPI Server did not provide any hint for a GPI linkage.

\section{Expression of putatively membrane-anchored equine CEACAMs}

As previously described, we have designed gene specific primer pairs of which the forward primer was located in the leader exon and the reverse primer in the $\mathrm{N}$ domain 

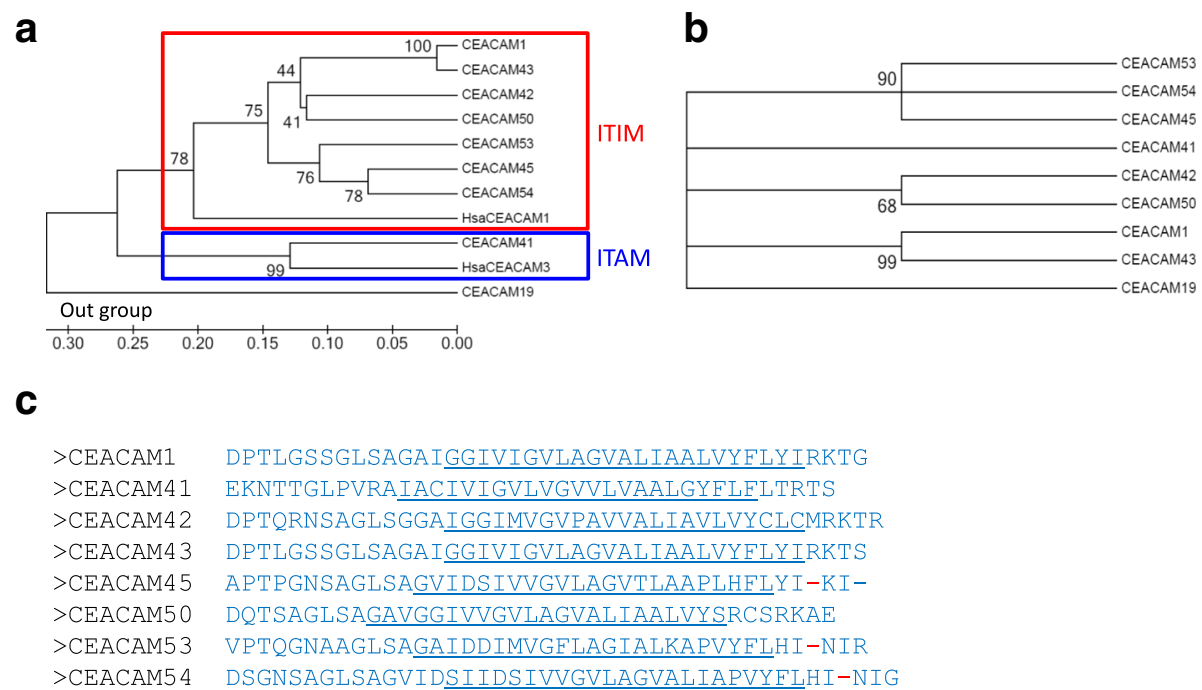

Fig. 1 Comparison of equine CEACAM TM domains. Phylogenetic trees were constructed from transmembrane domain exon nucleotide sequences (a), and amino acid sequences (b) using the UPGMA (a) and ML (b) method (MEGA 6.0 software). The reliability of a phylogenetic tree was assessed using the Bootstrap test applying 500 replicates. The statistical support for selected nodes is shown. Boxes group CEACAMs with the indicated properties. Note that on amino acid level the association of the TM with cytoplasmic signaling motifs is not visible. The transmembrane domain of Equine CEACAM19 was used as an out group. c Amino acid sequences of transmembrane domains of equine CEACAM-1 related CEACAMs. Predicted sequence of transmembrane helices by the TMHMM Server v. 2.0 http://www.cbs.dtu.dk/services/TMHMM-2.0/ are underlined. Stop codons within the transmembrane domain exon are indicated in red. Amino acids are depicted in single letter code. TM, transmembrane domain; CC, CEACAM; ITAM, immunoreceptor tyrosine-based activation motif; ITIM, immunoreceptor tyrosine-based inhibition motif

exon, respectively [15]. Using these primers, we performed a comprehensive analysis of gene expression in a set of horse tissues and cells (Tables 1 and 2). Various parts of the horse intestine were hot spots of membrane-bound CEACAM expression. In addition, in the mucosa of the vulva expression of several membrane-bound CEACAMs was found. CEACAM1, CEACAM43 and CEACAM50 were expressed in the liver. CEACAM42 and CEACAM43 were expressed in the kidney. Surprisingly, only CEACAM45 was found to be expressed in the spleen and no

Table 1 Expression of membrane anchored equine CEACAMs in various tissues of the horse

\begin{tabular}{|c|c|c|c|c|c|c|c|c|c|}
\hline Tissue & CC1 & CC19 & CC41 & CC42 & CC43 & CC45 & CC50 & CC53 & CC54 \\
\hline Salivary gland & & & & & & & & & + \\
\hline Tongue & & ++ & & & & & & & ++ \\
\hline Oesophagus & & ++ & & & & & & ++ & + \\
\hline Liver & +++ & & & & ++ & & + & & \\
\hline Duodenum & ++ & & & ++ & +++ & +++ & ++ & & ++ \\
\hline Jejunum & & & & + & ++ & ++ & & & \\
\hline lleum & & & & +++ & ++ & ++ & +++ & & ++ \\
\hline Caecum & + & & & ++ & + & +++ & ++ & & +++ \\
\hline Colon & & & & ++ & + & ++ & + & & ++ \\
\hline Rectum & + & & & ++ & + & ++ & & & \\
\hline Trachea & & & & & & & & ++ & ++ \\
\hline Lung & & & & & + & & & & \\
\hline Kidney & & & & + & ++ & & & & \\
\hline \multicolumn{10}{|l|}{ Bladder } \\
\hline Vulva & + & & & + & & +++ & & ++ & +++ \\
\hline Spleen & & & & & & + & & & \\
\hline
\end{tabular}


Table 2 Expression of membrane anchored equine CEACAMs in naïve and IL-2 stimulated immune cells

\begin{tabular}{|c|c|c|c|c|c|c|c|c|c|}
\hline Cell type & CC1 & CC19 & CC41 & CC42 & CC43 & CC45 & CC50 & CC53 & CC54 \\
\hline PBMC & & & & & & + & & & \\
\hline PBMC 7d_IL2 & ++ & & ++ & + & +++ & ++ & & & \\
\hline PBMC 14d_IL2 & & & ++ & & + & ++ & & & \\
\hline PBMC 19d_IL2 & & & ++ & & + & +++ & & & \\
\hline Granulocytes & +++ & & $t^{a}$ & & ++ & & & & \\
\hline
\end{tabular}

expression could be shown for CEACAM41 in tissues analyzed. Since several CEACAMs in humans are considered to play a pivotal role in the immune system, we further investigated CEACAM expression in white blood cells either unstimulated or after the stimulation with $500 \mathrm{U} / \mathrm{ml}$ IL-2. As shown in Table 2 granulocytes expressed CEACAM1, CEACAM41 and CEACAM43 while unstimulated PBMC expressed only CEACAM45. However, upon stimulation with IL-2, PBMC were found to express in addition to CEACAM45, also CEACAM1, CEACAM43 and CEACAM41 (Table 2).

\section{Alternative splicing (AS) of equine CEACAM mRNAs}

Diversity of CEACAM proteins was found to be enhanced by differential splicing in various animal species. Since nothing is known about the splicing of equine CEACAMs, we designed primers which were predicted to allow the amplification of full length cDNAs (Table 3). Tissues and cells in which strong expression was detected were selected for the amplification of full length cDNAs. cDNAs were isolated from the gel, cloned into cloning vectors and sequenced. All identified CEACAM transcripts are schematically depicted in Fig. 2. CEACAM1 was amplified from granulocytes and four different transcripts were identified. The extracellular part of the molecules contains an $\mathrm{N}$ domain and 0, 1 or three IgC-like domains. Specifically, we have cloned CEACAM1-4 L, CEACAM1-2 L, CEACAM12S and CEACAM1-1 L. The long isoforms harbor an ITIM and an ITSM in its cytoplasmic tail. CEACAM43 was amplified from the cDNA of the kidney and only one transcript composed of 4 extracellular Ig domains and an ITIM/ITSM containing cytoplasmic tail (CEACAM43-4 L) was present. Full length transcripts of CEACAM41 in sufficient amount for further analysis were only found in activated PBMC. Two splice variants were identified differing by the content of sequence coding for the cytoplasmic tail. However, none of CEACAM41 transcripts contain the transmembrane exon sequence. Thus, both transcript variants code for the same protein, a secreted molecule without ITAM motif. Transcripts of CEACAM42 were amplified from mRNA of the Caecum, since in different tissues from the small and large intestine a single full length transcript of around 1100 bp was amplified. The isolated transcript lacks sequences from the B-domain exon and from the $\mathrm{C} 1$ exon. Thus, CEACAM42 is a transmembrane molecule with two extracellular Ig-domains and a short cytoplasmic domain. CEACAM45 transcripts contain the cytoplasmic $\mathrm{C} 2$ and $\mathrm{C} 3$ exons. However, these two exons do not belong to the coding sequence since a stop codon is located in the transmembrane domain which is included in both identified transcripts. The two transcripts of CEACAM45 differ in their extracellular part; the major isoform has 3 Ig-domains while the minor isoform contains 2 Ig-domains. The transcript of CEACAM50 did neither contain the B-domain exon nor the cytoplasmic exons $\mathrm{C} 1$ and $\mathrm{C} 2$. Thus CEACAM50 has also a short cytoplasmic tail. The transcript coding for CEACAM53 included all exons of the gene. CEACAM54 has two unusual transcripts the first have an extended TM domain and the second did not include the TM domain.

\section{CEACAM42 has an extended transmembrane domain exon containing a stop codon}

In CEACAM42 the cytoplasmic exon 1 has a mutated splice donor site indicating that the cytoplasmic tail does not encode the ITIM/ITSM motifs. Indeed the structure of CEACAM42 mRNA demonstrated that the exon C1 is not included in the mRNA (Fig. 3a). Furthermore, there is also an alternative splice donor site at the end of the transmembrane domain exon. Thus, the transmembrane exon is extended by 37 nucleotides including a stop codon (Fig. 3b). Therefore the cytoplasmic exons 2 and 3 are not part of the coding sequence. The usage of the alternative splice donor site at the end of the transmembrane exon results in a transmembrane molecule with a short cytoplasmic tail of 11 amino acids. This short cytoplasmic tail contains a putative protein kinase A (PKA) phosphorylation site and a tyrosine-based sorting signal motif as predicted by the ELM software (Fig. 3c). In the intestine which is the main tissue of CEACAM42 expression (Table 1), only one transcript variant is expressed as indicated by a single band upon amplification of the full length cDNA (Fig. 3d). 
Table 3 Gene-specific oligonucleotides for expression analyses and CDNA cloning of horse CEA gene family members

\begin{tabular}{|c|c|c|c|}
\hline Gene & Oligonucleotide sequence & Location of primers (exon) & Size of PCR product (bp) \\
\hline \multirow[t]{2}{*}{ CEACAM1 } & For: TGCATCATATAAGATAGGCCCAG & $\mathrm{N}$ domain & 367 \\
\hline & Rev: AGTGAGAGTCCTCTTGTCCAGG & A1 domain & \\
\hline \multirow[t]{2}{*}{ CEACAM41 } & For: CATTGCATGTGATAGAGCGAC & $\mathrm{N}$ domain & 246 \\
\hline & Rev: CGTCCTTCTGTTCTGTGACTGT & A2 domain & \\
\hline \multirow[t]{2}{*}{ CEACAM42 } & For: AGGGGAAGGAATAGATCCCG & $\mathrm{N}$ domain & 392 \\
\hline & Rev: GAGTCCTGTTGTCCGAGGATAG & A1 domain & \\
\hline \multirow[t]{2}{*}{ CEACAM43 } & For: CCCATCAAGAAATTGTGTCCT & $\mathrm{N}$ domain & 276 \\
\hline & Rev: ATGTTAACACTACAGGGTCCCTG & A1 domain & \\
\hline \multirow[t]{2}{*}{ CEACAM44 } & For: GCTGTTGTAGGGACCGATGTTA & $\mathrm{N}$ domain & 503 \\
\hline & Rev: CCTCCTTCCTGATGATGTGTGT & A2 domain & \\
\hline \multirow[t]{2}{*}{ CEACAM45 } & For: GCGATAGGGCAACAAGAAATTAT & $\mathrm{N}$ domain & 254 \\
\hline & Rev: CATGTTAACACTACAGGGTCCTCA & A1 domain & \\
\hline \multirow[t]{2}{*}{ CEACAM46 } & For: AGTCCCACCCAATGGTATCC & $\mathrm{N}$ domain & 525 \\
\hline & Rev: CCCAAGTATTGCCCCTTCTGT & A1 domain & \\
\hline \multirow[t]{2}{*}{ CEACAM47 } & For: ACAGACCAAGTCCCAAAACC & $\mathrm{N}$ domain & 256 \\
\hline & Rev: TTACGTCTITTGACGATTCCAG & A1 domain & \\
\hline \multirow[t]{2}{*}{ CEACAM48 } & For: GACCAGCTCGCAAACAAA & $\mathrm{N}$ domain & 279 \\
\hline & Rev: AACAAGCTTCTTATCTGGCATTT & A2 domain & \\
\hline \multirow[t]{2}{*}{ CEACAM49 } & For: TGGAGCACGTCCACATAAAC & $\mathrm{N}$ domain & 232 \\
\hline & Rev: GGAGGTATTTGACCCTGGATT & A2 domain & \\
\hline \multirow[t]{2}{*}{ CEACAM50 } & For: AGATGCTCTTGAAGGAACGGAT & $\mathrm{N}$ domain & 453 \\
\hline & Rev: GACAGCTTCAGCCAGGTCCTA & A1 domain & \\
\hline CEACAM51 & ND & NA & NA \\
\hline \multirow[t]{2}{*}{ CEACAM52 } & For: ATGCTGCTGCAGGGGATA & $\mathrm{N}$ domain & 508 \\
\hline & Rev: CATCCTCCCTCCTGACACAT & A2 domain & \\
\hline \multirow[t]{2}{*}{ CEACAM53 } & For: TTCAAAGGGGAAATAGATTCCA & $\mathrm{N}$ domain & 394 \\
\hline & Rev: GAGTCCTGTTGTCTGGGGAC & A1 domain & \\
\hline \multirow[t]{2}{*}{ CEACAM54 } & For: ATCAGTCCCTGGCTTCAGA & $\mathrm{N}$ domain & 409 \\
\hline & Rev: TACACGGAGCTGTATACTTC & A domain & \\
\hline \multirow[t]{2}{*}{ CEACAM55 } & For: CCCTACTAGTCACGAGGAAGAAC & $\mathrm{N}$ domain & 222 \\
\hline & Rev: CATCCTCTCGGTCAGTCACA & A2 domain & \\
\hline \multirow[t]{2}{*}{ CEACAM1 } & For: GTCAGTAAGCTTCAGCAGACACCATGGAACTC & $5^{\prime} \cup T R$ & 1624 \\
\hline & Rev: GTCAGTTCTAGACAGTGAACAGGGCAGGATATG & $3^{\prime} U T R$ & \\
\hline \multirow[t]{2}{*}{ CEACAM41 } & For: GAGCAGTGCTTGTGAGCATT & $5^{\prime} \cup T R$ & 1131 \\
\hline & Rev: TAAGGGGAAGTTCCTGAAGG & $3^{\prime} U T R$ & \\
\hline \multirow[t]{3}{*}{ CEACAM42 } & For: TCACAGAGGGAGGGACAGAG & $5^{\prime} U T R$ & \\
\hline & Rev: GGGTAAAGGGATCCTTCCAG & $3^{\prime} U T R$ & 1390 \\
\hline & Rev: TCACAGAGGGAGGGACAGAG & TM domain & 1121 \\
\hline \multirow[t]{2}{*}{ CEACAM43 } & For: GTCAGTAAGCTTGACAGAGCAGGCAGCAGAC & $5^{\prime} \cup T R$ & 1613 \\
\hline & Rev: GTCAGTTCTAGAGAACAGGGCAGGTTGCAT & $3^{\prime} U T R$ & \\
\hline \multirow[t]{3}{*}{ CEACAM45 } & For: TCACAGAGGAAAGGACAGAGC & $5^{\prime} \cup T R$ & \\
\hline & Rev: CCTTGATTCCTGGACATTGAA & $3^{\prime} U T R$ & 1539 \\
\hline & Rev: CCTTGATTCCTGGACATTGAA & TM domain & 1379 \\
\hline CEACAM50 & For: CAGGAGTGCTTGTGAGAGTT & $5^{\prime} \cup T R$ & 1139 \\
\hline
\end{tabular}


Table 3 Gene-specific oligonucleotides for expression analyses and cDNA cloning of horse CEA gene family members (Continued)

\begin{tabular}{|c|c|c|c|}
\hline Gene & Oligonucleotide sequence & Location of primers (exon) & Size of PCR product (bp) \\
\hline & Rev: AGGGAAGAGGCTTCGTCTTC & 3'UTR & \\
\hline \multirow[t]{2}{*}{ CEACAM52 } & For: GTCAGTAAGCTTGCAGAAGCTCATCTCACAGAG & $5^{\prime} \mathrm{UTR}$ & 583 \\
\hline & Rev: GTCAGTTCTAGATTGTGAGGCAGATCAGATCC & $3^{\prime} U T R$ & \\
\hline \multirow[t]{2}{*}{ CEACAM53 } & For: CAGAGGGAGGGACAGAACAG & $5^{\prime} U T R$ & 1163 \\
\hline & Rev: ATGGCAGTTAGCCTTGGAGA & $3^{\prime} U T R$ & \\
\hline \multirow[t]{2}{*}{ CEACAM54 } & For: TCTCACAGAGGGAGGAGCCA & $5^{\prime} \cup T R$ & 916 \\
\hline & Rev: AGTCAGCAGTGCAGGAAACA & 3'UTR & \\
\hline
\end{tabular}

ND not done, NA not applicable, For forward primer, Rev. reverse primer

\section{CEACAM50 has a unique signaling motif in the cytoplasmic tail}

$C E A C A M 50$ has three cytoplasmic domain exons very similar to cytoplasmic exons of CEACAM1. Therefore, it may be expected that CEACAM50 has immune tyrosinebased signaling motifs. However, we found only transcripts lacking exon $\mathrm{C} 1$ and $\mathrm{C} 2$ (Fig. 4a). Furthermore, due to the use of an alternative splice donor site the transmembrane domain is prolonged by 14 nucleotides (Fig. $4 \mathrm{~b}$ ). Thus, the new splice donor site induces a frame shift and therefore the amino acid sequence of the cytoplasmic domain exon C3 is changed (Fig. 4b, c). Scanning the cytoplasmic tail of CEACAM50 for canonical signaling motifs identified a (PKA) phosphorylation site at the end of the cytoplasmic tail (Fig. 4c). Transcripts of CEACAM50 were preferentially detected in tissues of the intestine (Fig. 4d). In order to confirm that CEACAM50 could be expressed at the cell surface we fused a FLAG-tag to the N-terminus of

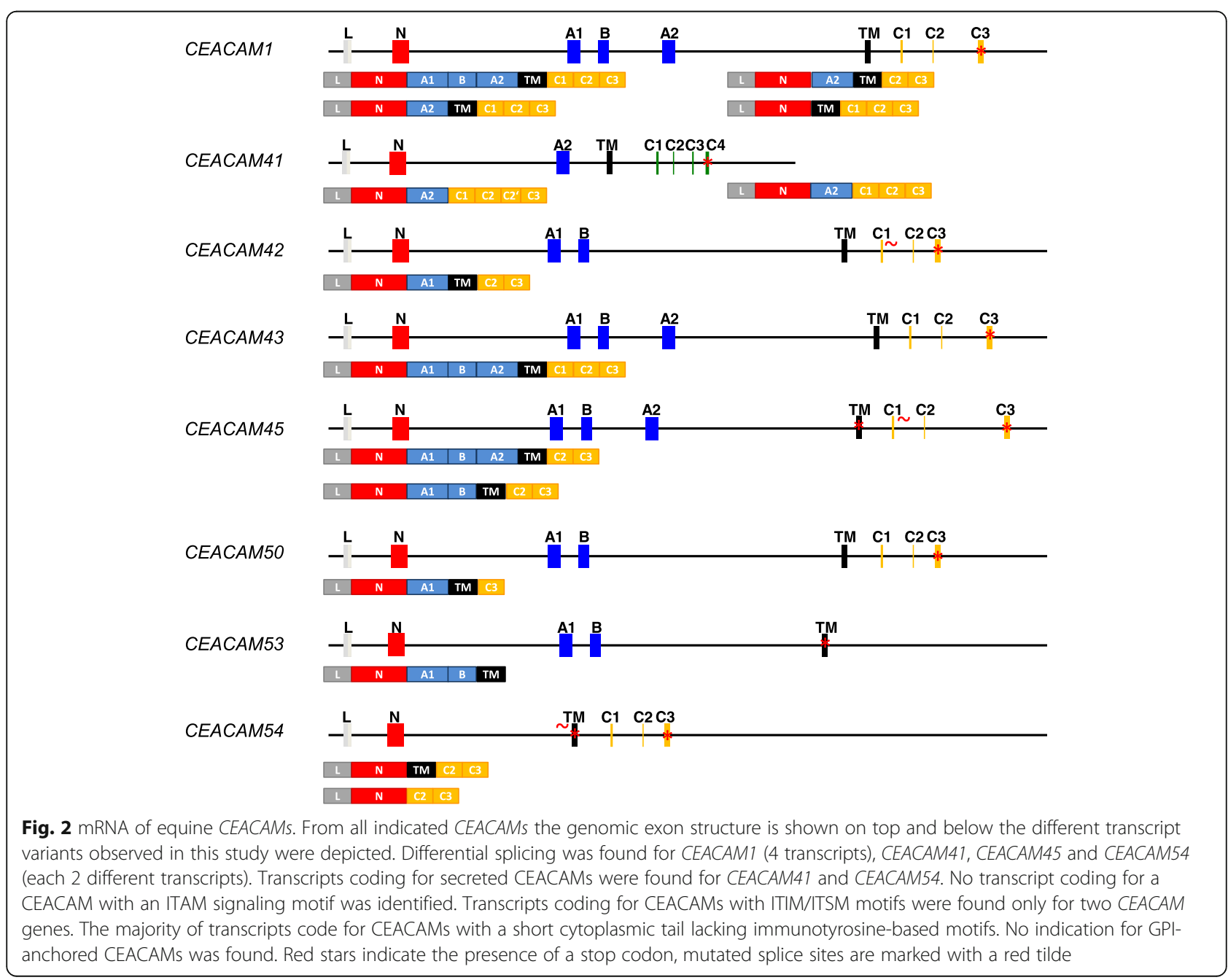




\section{a}

CEACAM42

CEACAM42

MRNA

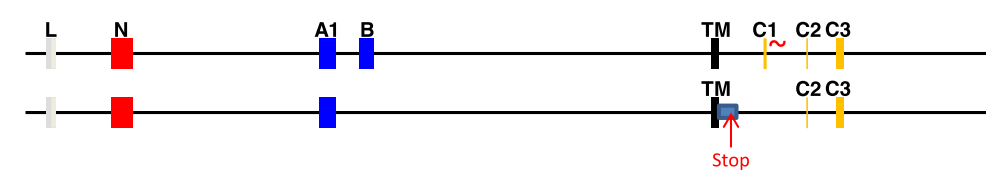

b

TM exon sequence

ATGATCCAACACAAAGAAATTCTGCTGGCCTCTCAGGTGGGGTCATTGGTGGCATCATGGTTGGA GTCCCAGCTGTGGTAGCTCTGATAGCGGTCCTGGTGTACTGCCTGTGTATGAGAAAGACTCGAGA GTATTATGGCCCTTTTTAGTGTTTTGCTTTCTCAAAGGTGAGC

Mutated splice site Stop codon $\quad$ Alternative splice donor site

C

TM

DPTQRNSAGLSGGVIGGIMVGVPAVVALIAVLVYCLCMRKTREYYGPF

Y-based sorting signal

PKA Phosphorylation site

d

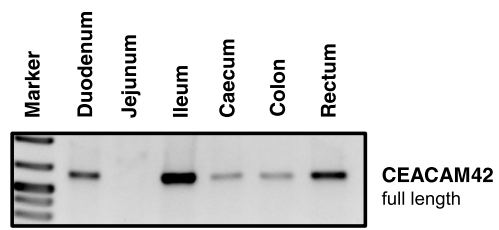

Fig. 3 CEACAM42 has an extended transmembrane domain. The exon structure of the CEACAM42 gene indicates that its gene product may contain an ITIM signaling motif. However as shown in a the cloned mRNA demonstrate that an alternative splice donor site of the transmembrane exon is used and that the exons C1 was not integrated into the mRNA. The stop codon which exists in the extended transmembrane exon is indicated by the red arrow and the "stop". The mutated splice donor sites of C1 is indicated with a red tilde. $\mathbf{b}$ shows the possible but unused splice donor site (underlined). c Amino acid sequence of the transmembrane and cytoplasmic part of CEACAM42. The PKA phosphorylation site is highlighted in blue, the predicted Y-based sorting signal in red. $\mathbf{d}$ Expression of CEACAM42 was detected in various parts of the intestine

CEACAM50 and expressed the fusion protein in Cos7L cells. As shown in Fig. 4e the staining of living transfected cells with anti-Flag antibodies by flow cytometry strongly indicates that the fusion protein is located at the cell surface. Thus, CEACAM50 is a transmembrane CEACAM with a unique signaling motif not found in other species.

\section{CEACAM54 has a unique membrane proximal extracellular structure}

The CEACAM54 gene consists of six exons (Fig. 5a), the transmembrane exon and the $\mathrm{C} 3$ exon contains stop codons. The canonical splice acceptor site of the transmembrane exon is disrupted, due to the insertion of a simple sequence repeat (SSR) (Fig. 5a and b). An alternative splice acceptor site is located in front of the inserted simple sequence repeat. Indeed as demonstrated by cDNA cloning, two transcript variants were identified. The first variant contains the transmembrane exon including the SSR, whereas in the second transcript the transmembrane exon, including the SSR, and the C1 exon are excluded (Fig. 5a). The first transcript variant has an open reading frame until the stop codon at the end of the transmembrane exon. The SSR codes for a Proline, Threonine and Arginine-rich extracellular membrane proximal region.
CEACAM54 mRNA was detected in the intestine, trachea and vulva. Interestingly, while in most tissues transcript variant 1 was dominant, in the vulva mucosa variant 2 was prominent (Fig. 5c). Again we tested if the mRNA is translated into protein and if this protein is expressed at the cell surface. As shown in Fig. 5d and e Flag-tagged CEACAM54 is expressed at the cell surface of transfected Cos7L cells.

\section{Loss of membrane-anchorage and signaling via an ITAM of CEACAM41}

CEACAM41 gene is the only equine CEACAM that harbors exons which may code for an ITAM in the cytoplasmic tail similar to that found in human CEACAM3 and CEACAM4. In humans, ITAM harboring CEACAMs are expressed specifically in granulocytes $[18,19]$. However, we did found CEACAM41 transcripts only in granulocytes isolated from one out of three horses. In addition, we did not find CEACAM41 transcripts in any other tissue we have analyzed. Since expression of some CEACAMs, i.e. CEACAM1 by $\mathrm{T}$ cells is activation dependent we analyzed expression of CEACAM41 by stimulated equine PBMC (Fig. 6a). Amplification of full length CEACAM41 cDNA reveals that two different transcripts are 
a

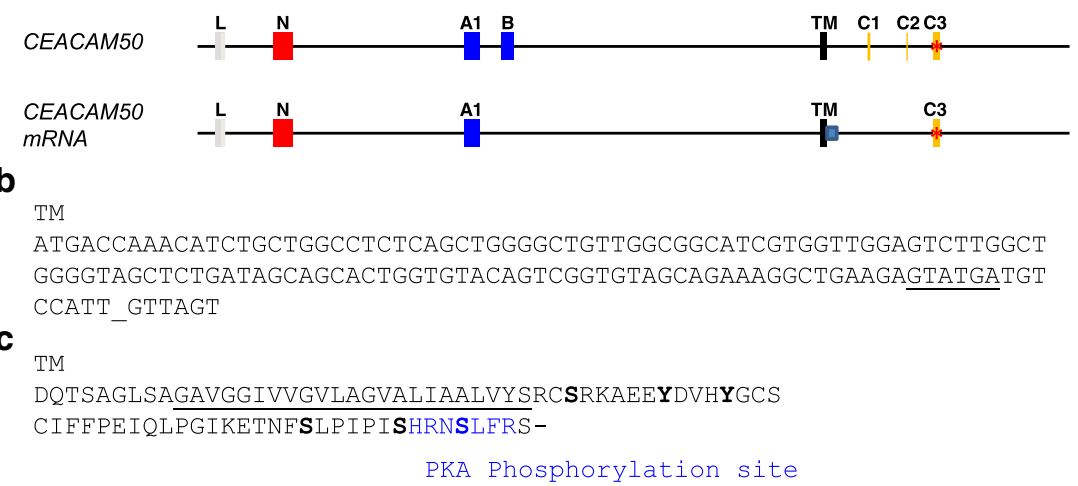

d
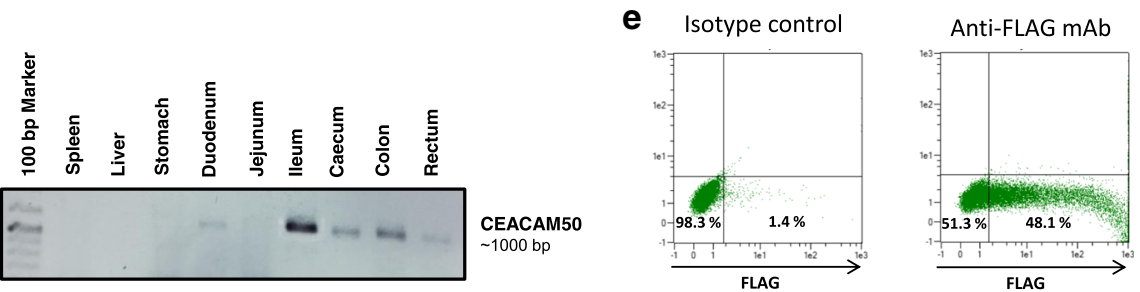

Fig. 4 CEACAM50 has unique signaling motifs within the CEA gene family. The exon structure of the CEACAM50 gene indicates that its gene product may contain an ITIM signaling motif. However as shown in a the cloned mRNA demonstrate that an alternative splice donor site of the transmembrane exon is used and that the exons $\mathrm{C} 1$ and $\mathrm{C} 2$ were not integrated into the mRNA. $\mathbf{b}$ shows the possible but unused splice donor site (underlined). c Amino acid sequence of the transmembrane and cytoplasmic part of CEACAM50. The PKA phosphorylation site is highlighted in blue, further phosphorylation sites were indicated in bold. $\mathbf{d}$ Expression of CEACAM50 was detected in various parts of the intestine. e Expression of CEACAM50 fused to a FLAG-tag at the N-terminus by transfected Cos7L. Red stars indicate the presence of a stop codon

generated in IL-2 activated PBMC (Fig. 6b). Cloning and sequencing of both cDNAs demonstrated that in both transcripts the transmembrane exon is excluded leading to a frame shift and a new stop codon at the $5^{\prime}$-end of the extended $\mathrm{C} 1$ exon (Fig. 6c). Thus, both transcripts code for one protein which consist of one IgV-like and one IgC-like domain followed by a short peptide (Fig. 6d). The short peptide has no sequence similarity to the predicted cytoplasmic sequence of the genomic exon sequence containing an ITAM-like motif (Fig. 6e).

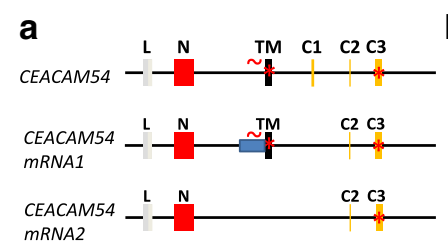

b Insertion

CAACAACAACACCAAGACCAACACCAACACCAACACCAAGACCAACACCAACACCAACACCAAG ACCAACACCAACACCAAGACCAACACCAACACCAACGCCAAGACCAACACCAAGACCAACACCA ACCAACACCAACACCAAGACCAACACCAACACCAACGCCAAGACCAACACCAAGACCAACACCA

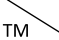

DSTTTPRPTPTPTPRPTPTPTPRPTPTPRPTPTPTPRPTPRPTPTPTPTPTPGNSAGLSA GVIDSIIDSIVVGVLAGVALIAPVYFLHI-
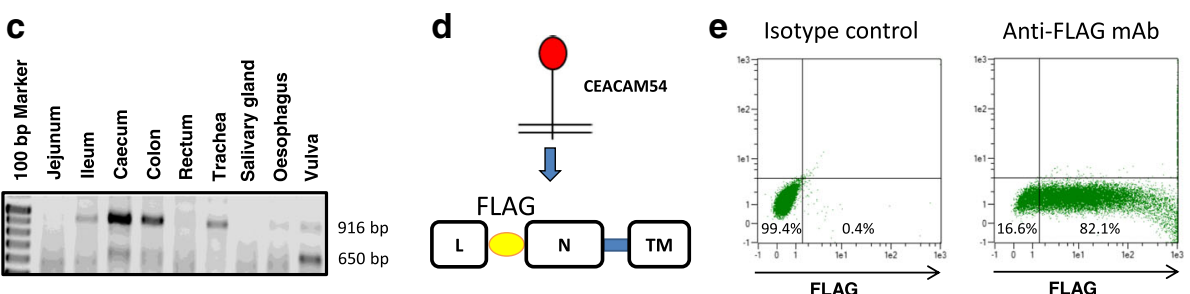

Fig. 5 CEACAM54 contains a P-T-R rich membrane proximal extracellular region. a Exon structure of the CEACAM54 gene and the two transcript variants identified in the present study. The simple sequence inserted in front of the transmembrane domain and the translation into the amino acid sequence (blue box) are depicted in (b). c Expression of transcript variant 1 (916 bp) in the intestine and trachea and of variant 2 (650 bp) in the vulva mucosa. Cartoon of the Flag-tag-CEACAM54 fusion protein (d). Expression of the Flag-tagged CEACAM54 at the cell surface of Cos7L cells as detected by flow cytometry (e). Red stars indicate the presence of a stop codon, mutated splice sites are marked with a red tilde 


\section{a}

PBMC $(500 \mathrm{U} / \mathrm{ml}$ rhlL-2)

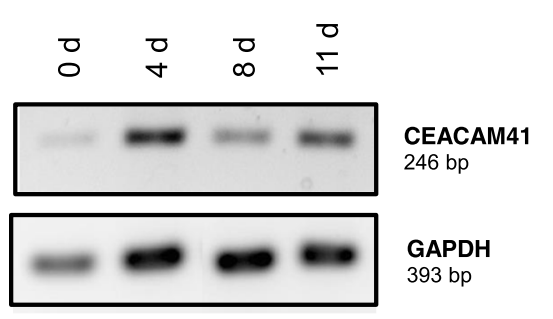

C

Transcript 1

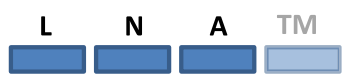

Transcript 2

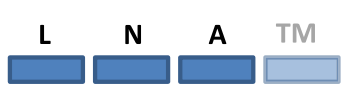

e

\section{Exon prediction}

RTRGQPDLTEHRPPASSPGRGSSSSSASPAPLPGSRTVIPIYQELLHPHANTYCQINHKADVAS

\section{Transcript}

SLVEKGGLHPLLGSEDSLCPLF-

Fig. 6 CEACAM41 is a secreted protein. a PBMC were cultured with $500 \mathrm{U} / \mathrm{ml}$ rhlL-2 for the indicated times. RT-PCR using primers located in the leader and the $\mathrm{N}$-domain suggested that CEACAM41 is preferentially expressed by stimulated PBMC. Full length amplification of CEACAM41 CDNA identified two different transcripts $(\mathbf{b})$. The two transcripts differ from each other by a short sequence, inserted between exon C2 and C3 further named $\mathrm{C2}^{\prime}$ exon (c). The stop codon which exists in the extended C1 exon is indicated by the red arrow and the "stop" (c). Both transcripts code for the same protein (d). e Comparison of the amino acid sequence of the cytoplasmic tail of CEACAM41 as predicted from the exon sequence of the CEACAM41 gene and the peptide sequence encoded by the cytoplasmic exons in transcripts without transmembrane domains

\section{Discussion}

Gene duplication (GD) and alternative splicing (AS) are the two main mechanisms responsible for functional protein diversity [20, 21]. GD generates a significant expansion of the CEA gene family in various mammalian species resulting in up to 100 different CEACAM genes in certain bat species [9]. Protein diversity in the CEA family is further enhanced by extensive AS of certain CEACAM mRNAs, for example human CEACAM1 codes for 12 different proteins [22, 23]. A more sophisticated interplay between GD and AS is the possibility to generate functional diversity of duplicated genes by AS [24]. Variation of AS between the ancestral gene and the duplicated gene may favor the fixation of the duplicate, due to new functional properties gained by AS. Indeed, it was observed that duplicated genes have a reduced amount of AS events per gene compared with the ancestral gene [24]. Such a reduction of AS in duplicated genes may change their function in the context of a specific cell type or tissue were they are expressed. Consistent with this hypothesis we only found a single splice isoform for CEACAM43 while four isoforms of CEACAM1 were detected. Surprisingly, AS was not found to play a major role for functional diversification of $C E A$ CAM1 paralogs in CEA gene families so far investigated in more detail. However, the discrepancy between the predicted structure of equine CEACAMs and the sequenced mRNAs suggests that AS is a pivotal mechanism for CEACAM1 paralog diversification in the horse and most likely in other mammals. However, we would like to point out that the absence of finding particular splice variants, does not mean that these protein variants are not expressed anywhere in the horse tissues, since both tissue distribution and cellular activation state are known to influence processing of the transcripts from the CEA family. AS effects primarily the transmembrane domain exon, either at the $5^{\prime}$ end or the $3^{\prime}$ end. The reason may be that modifications at the transmembrane domain have a high probability to change the function of the duplicated gene product by means of modifying signal transduction $[25,26]$. Four out of seven equine CEACAM1 paralogs have gained new signaling motifs 
by AS. For example, CEACAM42 and CEACAM50 mRNA were modified by using an alternative splice donor site at the transmembrane exon and to ignore splice acceptor sites of the first and second (only CEACAM50) cytoplasmic exons. In both cases the potential ITMS/ITIM motifs were eliminated. An AS of CEACAM54 mRNA leads to two transcripts, one having a very unique proline-threonine-arginine-rich domain encoded by a SSR at the membrane proximal part of the extra cellular region, and the second missing a transmembrane region. Since the P-T-R-rich domain is not found in any other mammalian protein in the NCBI-data bases we argued that this protein could be expressed. However, we were able to express FLAG-tagged CEACAM54 on the cell surface of Cos7 cells by transient transfection, indicating that CEACAM54 is expressed in the horse. Taken together, only CEACAM1 and CEACAM43 retained the inhibitory signaling motifs. This is similar to the CEA gene family in mice which also contain two inhibitory CEACAMs [27]. And also the different expression pattern of inhibitory CEACAMs was previously observed for murine CEACAM1 and murine CEACAM2 [10].

One reason for the restricted number of inhibitory CEACAMs may be that CEACAM1 is a checkpoint molecule for $\mathrm{T}$ and $\mathrm{B}$ cell activation, and therefore an increase of the gene dosage, after GD, may be fatal to a wellbalanced regulation of immune responses [28]. Similar observations were made in the Signaling regulatory protein (SIRP) family. Although the founder gene is the inhibitory receptor SIRPa [29], the expanded SIRP families comprise only a single ITIM containing receptor in all investigated species [30]. The authors suggested that this might be consistent with a homeostatic function of SIRP $\alpha$, like recognizing "self" in the form of the broadly expressed surface marker $\mathrm{CD} 47$, which negatively regulate the function of innate immune cells, such as macrophages [30]. The number of activation receptors is not limited like the inhibitor receptors in families containing paired receptors like the CEA, SIRP and natural killer cell inhibitory receptor (KIR) gene families [13, 30, 31].

Furthermore, and in certain aspects the most striking modification induced by AS was observed for CEACAM41 mRNA. Both transcripts code for the same amino acid sequence lacking a transmembrane domain. The GPI anchored CEACAMs in primates have a stop codon within the transmembrane domain which shortens the transmembrane helix in the way that they do no longer have a charged amino acid at the cytoplasmic border of the cell membrane, thus it is not properly fixed in the cell membrane. On the other hand the residual transmembrane domains provide the necessary signals for the GPI anchorage. In the horse the secreted CEACAM41 did not contain any part of the transmembrane domain, thus also lacking a GPI signal. This indicates that CEACAM41 is not attached to the cell membrane, but it is secreted. Thus no activating CEACAM exists in the horse. This is again similar to the murine CEA family which does not contain activating CEACAMs [32]. From an evolutionary point of view, the presence of a putative activating CEACAM receptor gene which still has intact exons and splice sites indicates that a functional activating receptor in the horse existed until recently. One may speculate that at a certain point of equine history the selective pressure, putatively a pathogen, for the activating CEACAM got lost. From that time point on the activating CEACAM was free to change his function or to get eliminated from the genome. AS which renders the activating receptor into a secreted molecule would change the function of the molecule both rapidly and fundamentally. Once a completely new CEACAM is created selection may search for an optimized spatio-temporal expression pattern for the new function. This period of selection may have led to the expression of CEACAM41 by activated PBMC. Remarkably, CEACAM41 is not the only secreted CEACAM expressed by equine activated PBMC, since CEACAM46a and CEACAM46b are also secreted by PBMC upon activation [15]. Comparing the ligand binding domain ( $\mathrm{N}$-domain) of other secreted equine CEACAMs showed that CEACAM44 and CEACAM55 cluster together with that of CEACAM46a and CEACAM46b suggesting that they may share common ligands [15]. It is well known that hemophilic interaction of membrane bound CEACAMs regulate the activation of immune cells in trans [33, 34]. Furthermore it was described that soluble CEACAMs interact with membrane-bound CEACAMs in a homophilic and heterophilic fashion [35-37]. Thus it is tempting to speculate that equine lymphoid cells secrete CEACAMs upon activation in order to transmit regulatory signals to distantly located immune cells. Putative ligands of the secreted CEACAMs on immune cells may include CEACAM1, CEACAM43 and CEACAM45. Together these considerations suggest that equine lymphoid cells have acquired a novel mechanism based on the secretion of CEACAMs to regulate an immune response. Further investigations are required to substantiate secreted CEACAMs could be useful therapeutic targets to modulate immune responses in the horse.

\section{Conclusion}

Gene family expansion is a potent evolutionary process to adapt to environmental cues. In most cases gene duplication is accompanied by sequence diversification of paralogues genes. Recently we have identified that in certain bat species the ligand-binding domain of CEACAMs is under positive selection. In the present report we show, that in the horse a second mechanism of gene diversification is active. AS after gene duplication, that preferentially affect cell membrane anchorage and the 
cytoplasmic tail of CEACAM1 paralogs, is most likely a mechanism that may rapidly change the functional properties of the paralogous gene by changing its signaling capacity. Such potent mechanisms of gene variation may extraordinarily accelerate adaption to environmental cues. It is intriguing that such a mechanism is involved in the evolution of a gene family which is thought to be part of host-pathogens arms race.

\section{Methods}

\section{Cells and tissues}

Different equine tissue samples were collected from freshly slaughtered healthy horses and either flash-frozen in liquid nitrogen or stored in RNAlater (Invitrogen). Peripheral blood mononuclear cells (PBMCs) and granulocytes were isolated from blood of healthy horses by density-gradient centrifugation through Ficoll-Paque 1077 g/l (GE Healthcare). Stimulation of PBMC with human rIL-2 (Proleukin, Chiron) was performed with $200 \mathrm{U} / \mathrm{ml}$ for the indicated time, at a concentration of $5 \times 10^{5}$ cells $/ \mathrm{ml}$ in RPMI-1640 supplemented with $10 \%$ fetal calf serum (FCS "Gold"; Bio\&SELL), $2 \mathrm{mM}$ L-glutamine, $100 \mathrm{U} / \mathrm{ml}$ penicillin, $100 \mu \mathrm{g} / \mathrm{ml}$ streptomycin, non-essential amino acids and $1 \mathrm{mM}$ sodium pyruvate (GIBCO/Invitrogen). Magnetic cell separation (Miltenyi Biotec) was used for the isolation of lymphocyte subtypes. CD4 and CD8 positive cells were isolated with murine IgG1 primary mAb (compare "Cell transfection and flow cytometry" below) and anti-mouse IgG MicroBeads.

\section{Identification and prediction of equine CEACAMs}

Equine CEACAMs were identified similar to the method described previously [9]. For sequence similarity searches we used the NCBI BLAST tools "blastn" http://blast.ncbi.nlm.nih.gov/Blast.cgi and Ensembl BLAST/BLAT search programs http://www.ensembl.org/Multi/Tools/Blast?db=core using default parameters. For identification of horse $C E A$ $C A M$ exons, exons from known CEACAM and PSG genes from other species were used to search "whole-genome shotgun contigs (wgs)" databases limited to organism "Equus caballus (taxid:9796)". Hits were considered to be significant if the E-value was $<\mathrm{e}-10$ and the query coverage was $>50 \%$. Once a wgs contig containing CEACAMrelated sequences was identified we manually confirmed the presence of the complete exon according to its size and the presences of CEACAM-typical splice site sequences. The gene structure was predicted according to known CEACAMs. Predicted CEACAM genes were further compared with the horse genome Ensembl/NCBI release EquCab2.

\section{Expression analysis by reverse transcription-polymerase chain reaction}

RT-PCR was carried out as previously described [2]. In brief, total RNA was extracted using the RNeasy kit
(Qiagen). One microgram of total RNA was transcribed using AMV Reverse Transcriptase (Promega). The RT product was amplified with Taq polymerase (Fermentas). After denaturation at $95^{\circ} \mathrm{C}$ for $45 \mathrm{~s}, 35$ PCR cycles (denaturation: $95{ }^{\circ} \mathrm{C}, 30 \mathrm{~s}$; annealing: $60{ }^{\circ} \mathrm{C}, 1 \mathrm{~min}$; extension: $72{ }^{\circ} \mathrm{C}, 1.5 \mathrm{~min}$ ) and a final extension at $72{ }^{\circ} \mathrm{C}$ for 15 min were performed. Primers used were summarized in Table 3. Eight microliters of each PCR product were analyzed by electrophoresis on a 1.8\% agarose gel and visualized by GelRed (Biotium) staining.

\section{CDNA cloning}

RNA isolation an RT was performed as described for expression analysis. Primers used for amplification of a fulllength cDNAs are shown in Table 3. For cDNA cloning the RT product was amplified by polymerase chain reaction (PCR) with Easy-A High-Fidelity PCR Cloning Enzyme (Agilent) and analyzed by agarose gel electrophoresis. Specific bands were extracted from the agarose gel using QIAEX II Gel Extraction Kit (Qiagen). The PCR-products were cloned using the StrataClone PCR Cloning Kit (Agilent). Plasmid DNA isolated from various clones were analyzed by PCR and sequencing. Nucleotide sequencing was performed with the BigDye Terminator Cycle Sequencing Kit (PE Applied Biosystems).

\section{Cell transfection and flow cytometry}

For expression of equine CEACAMs by eukaryotic cells, full length cDNA was transferred from the StrataClone Cloning vector into the shuttle vector pFLAG-CMV3 (Sigma-Aldrich). $1 \times 10^{6}$ Cos7 cells were transfected using the Nucleofector Kit V (Amaxa). $1 \times 10^{5}$ transiently transfected Cos7 cells were stained with murine anti-FLAG $\mathrm{mAb}$ (clone M2, Sigma-Aldrich) as a primary mAb and using anti-mouse IgG-PE as secondary antibody (goat antimouse). Flow cytometry was performed with the MACSQuant Analyzer and the "MACS Quantify" software.

\section{Bioinformatics}

Phylogenetic analyses based on nucleotide and amino acid sequences were conducted using MEGA6. Sequences were aligned using "Muscle" and the maximum likelihood (ML) or unweighted pair group method with arithmetic mean (UPGMA) method with bootstrap testing (500 replicates) was applied for the construction of phylogenetic trees. Sequence motif identification was performed using the sequence pattern search program ELM (http://elm.eu.org/). Transmembrane helixes were identified using the TMHMM Server at http:// www.cbs.dtu.dk/services/TMHMM-2.0/ and GPI anchors were predicted by the PredGPI Server at http:// gpcr.biocomp.unibo.it/predgpi/. Phosphorylation sites were identified by the NetPhos 3.1 Server at http:// www.cbs.dtu.dk/services/NetPhos/. 


\section{Abbreviations}

AS: Alternative splicing; CEA: Carcinoembryonic Antigen;

CEACAM: Carcinoembryonic Antigen-related cell adhesion molecule; GD: Gene duplication; GPI: Glycosylphosphatidylinositol; ISF: Immunoglobulin super family; ITAM: Immunoreceptor tyrosine-based activation motif; ITIM: Immunoreceptor tyrosine-based inhibitory motif; ITSM: Immunoreceptor tyrosine-based switch motif; KIR: Killer cell inhibitory receptor; ML: Maximum likelihood; PBMC: Peripheral blood mononuclear cells; PCR: Polymerase chain reaction; PSG: Pregnancy-specific glycoproteins; RT: Reverse transcription; SIRP: Signaling regulatory protein; TM: Transmembrane; WGS: Whole-genome shotgun

\section{Acknowledgements}

We thank Andrea Braun, Franziska Thomas and Lisa Faust-Klüger for expert technical assistance.

\section{Funding}

This study was supported by the BMWi (KF2875802UL2), GIZ (Contract no. 81170269; Project No. 13.1432.7-001.00) and DFG (HE 6249/4-1) (to R.K.) This funding source had no role in the design of the study and collection, analysis, and interpretation of data and in writing the manuscript.

\section{Availability of data and materials}

Nucleotide sequences from equine CEACAMs are available at NCBI GenBank accession numbers MF564057- MF564071. Phylogenetic trees, sequence data and alignments used to produce the results are available at TreeBASE (http:// purl.org/phylo/treebase/phylows/study/TB2:S21963).

\section{Authors' contributions}

SM, performed most of the experiments and data analysis, DA, LB, TH, KYL, $\mathrm{JH} 1, \mathrm{MM}$, performed experiments, contributed to data analysis, $\mathrm{JH}$, , provided critical reagents, contributed substantially to data interpretation and critically revised the manuscript. RK conceived the study, carried out data analysis and drafted the manuscript. All authors contributed to manuscript writing, read and approved the final manuscript.

\section{Ethics approval and consent to participate}

Healthy horses were slaughtered for meat production at the abattoir "Beerwart, Waiblingen", not as part of this study, however we got permission from the abattoir to use of the tissues for the present study. Further tissue collection was approved by animal use committee of local authorities (Landesamt für Landwirtschaft, Lebensmittelsicherheit und Fischerei (LALLF) Rostock, Germany; 7221.3-2.1-011/13).

\section{Consent for publication}

Not applicable.

\section{Competing interests}

The authors declare that they have no competing interests.

\section{Publisher's Note}

Springer Nature remains neutral with regard to jurisdictional claims in published maps and institutional affiliations.

\section{Author details}

Institute of Immunology, Friedrich-Loeffler-Institut, Suedufer 10, Greifswald, Insel Riems, Germany. ${ }^{2}$ Plattform Degenerative Erkrankungen, Deutsches Primatenzentrum $\mathrm{GmbH}$, Goettingen, Germany. ${ }^{3}$ Department of Cellular Biochemistry, Max-Planck-Institute of Biochemistry, Martinsried, Germany. ${ }^{4}$ Department of Chemistry, Korea Advanced Institute of Science and Technology (KAIST), Daejeon, Republic of Korea. ${ }^{5}$ Clinic for Horses, Veterinary Faculty, Freie Universität Berlin, Oertzenweg 19b, D-14163 Berlin, Germany. ${ }^{6}$ Friedrich-Loeffler-Institut, Bundesforschungsinstitut für Tiergesundheit, Federal Research Institute for Animal Health, Südufer 10, D, 17493 Greifswald, Insel Riems, Germany.

\section{Received: 17 August 2017 Accepted: 2 March 2018}

Published online: 15 March 2018

\section{References}

1. Kammerer R, Hahn S, Singer BB, Luo JS, von Kleist S. Biliary glycoprotein (CD66a), a cell adhesion molecule of the immunoglobulin superfamily, on human lymphocytes: structure, expression and involvement in T cell activation. Eur J Immunol. 1998;28(11):3664-74.

2. Kammerer R, Popp T, Singer BB, Schlender J, Zimmermann W. Identification of allelic variants of the bovine immune regulatory molecule CEACAM1 implies a pathogen-driven evolution. Gene. 2004;339:99-109.

3. Adler H, El-Gogo S, Guggemoos S, Zimmermann W, Beauchemin N, Kammerer R. Perturbation of lytic and latent gammaherpesvirus infection in the absence of the inhibitory receptor CEACAM1. PLoS One. 2009;4(7):e6317.

4. Adler $\mathrm{H}$, Steer B, Juskewitz E, Kammerer R. To the editor: murine gammaherpesvirus 68 (MHV-68) escapes from NK-cell-mediated immune surveillance by a CEACAM1-mediated immune evasion mechanism. Eur J Immunol. 2014;44(8):2521-2.

5. Boulton IC, Gray-Owen SD. Neisserial binding to CEACAM1 arrests the activation and proliferation of CD4+ T lymphocytes. Nat Immunol. 2002;3(3):229-36

6. Sintsova A, Sarantis H, Islam EA, Sun CX, Amin M, Chan CH, Stanners CP, Glogauer M, Gray-Owen SD. Global analysis of neutrophil responses to Neisseria gonorrhoeae reveals a self-propagating inflammatory program. PLoS Pathog. 2014;10(9):e1004341.

7. Kammerer R, Zimmermann W. Coevolution of activating and inhibitory receptors within mammalian carcinoembryonic antigen families. BMC Biol. 2010;8:12.

8. Zimmermann W, Kammerer R. Coevolution of paired receptors in Xenopus carcinoembryonic antigen-related cell adhesion molecule families suggests appropriation as pathogen receptors. BMC Genomics. 2016;17(1):928.

9. Kammerer R, Mansfeld M, Hanske J, Missbach S, He X, Kollner B, Mouchantat $\mathrm{S}$, Zimmermann $\mathrm{W}$. Recent expansion and adaptive evolution of the carcinoembryonic antigen family in bats of the Yangochiroptera subgroup. BMC Genomics. 2017;18(1):717.

10. Han E, Phan D, Lo P, Poy MN, Behringer R, Najjar SM, Lin SH. Differences in tissue-specific and embryonic expression of mouse Ceacam1 and Ceacam2 genes. Biochem J. 2001;355(Pt 2):417-23.

11. Thompson JA. Molecular cloning and expression of carcinoembryonic antigen gene family members. Tumour Biol. 1995;16(1):10-6.

12. Naghibalhossaini F, Stanners CP. Minimal mutations are required to effect a radical change in function in CEA family members of the lg superfamily. J Cell Sci. 2004;117(Pt 5):761-9.

13. Kammerer R, Popp T, Hartle S, Singer BB, Zimmermann W. Species-specific evolution of immune receptor tyrosine based activation motif-containing CEACAM1-related immune receptors in the dog. BMC Evol Biol. 2007:7:196.

14. Weichselbaumer $M$, Willmann $M$, Reifinger $M$, Singer J, Bajna E, Sobanov $Y$, Mechtcherikova D, Selzer E, Thalhammer JG, Kammerer R, et al. Phylogenetic discordance of human and canine carcinoembryonic antigen (CEA, CEACAM) families, but striking identity of the CEA receptors will impact comparative oncology studies. PLoS Curr. 2011;3:RRN1223.

15. Aleksic D, Blaschke L, Missbach S, Hanske J, Weiss W, Handler J, Zimmermann W, Cabrera-Sharp V, Read JE, de Mestre AM, et al. Convergent evolution of pregnancy-specific glycoproteins in human and horse. Reproduction. 2016:152(3):171-84.

16. Moore T, Dveksler GS. Pregnancy-specific glycoproteins: complex gene families regulating maternal-fetal interactions. Int J Dev Biol. 2014;58 $(2-4): 273-80$

17. Pavlopoulou A, Scorilas A. A comprehensive phylogenetic and structural analysis of the carcinoembryonic antigen (CEA) gene family. Genome Biol Evol. 2014;6(6):1314-26.

18. Delgado Tascon J, Adrian J, Kopp K, Scholz P, Tschan MP, Kuespert K, Hauck CR. The granulocyte orphan receptor CEACAM4 is able to trigger phagocytosis of bacteria. J Leukoc Biol. 2015;97(3):521-31.

19. Nagel G, Grunert F, Kuijpers TW, Watt SM, Thompson J, Zimmermann W. Genomic organization, splice variants and expression of CGM1, a CD66-related member of the carcinoembryonic antigen gene family. Eur J Biochem. 1993; 214(1):27-35.

20. Stoffel A, Neumaier M, Gaida FJ, Fenger U, Drzeniek Z, Haubeck HD, Wagener C. Monoclonal, anti-domain and anti-peptide antibodies assign the molecular weight 160,000 granulocyte membrane antigen of the CD66 cluster to a mRNA species encoded by the biliary glycoprotein gene, a member of the carcinoembryonic antigen gene family. J Immunol. 1993; 150(11):4978-84

21. Pan $Q$, Shai O, Lee $L J$, Frey BJ, Blencowe BJ. Deep surveying of alternative splicing complexity in the human transcriptome by high-throughput sequencing. Nat Genet. 2008;40(12):1413-5. 
22. Barnett TR, Drake L, Pickle W 2nd. Human biliary glycoprotein gene: characterization of a family of novel alternatively spliced RNAs and their expressed proteins. Mol Cell Biol. 1993;13(2):1273-82.

23. Barnett TR, Kretschmer A, Austen DA, Goebel SJ, Hart JT, Elting JJ, Kamarck ME. Carcinoembryonic antigens: alternative splicing accounts for the multiple mRNAs that code for novel members of the carcinoembryonic antigen family. J Cell Biol. 1989;108(2):267-76.

24. Iniguez LP, Hernandez G. The evolutionary relationship between alternative splicing and gene duplication. Front Genet. 2017;8:14.

25. Eshel D, Toporik A, Efrati T, Nakav S, Chen A, Douvdevani A. Characterization of natural human antagonistic soluble CD40 isoforms produced through alternative splicing. Mol Immunol. 2008;46(2):250-7.

26. Dery KJ, Kujawski M, Grunert D, Wu X, Ngyuen T, Cheung C, Yim JH, Shively JE. IRF-1 regulates alternative mRNA splicing of carcinoembryonic antigenrelated cell adhesion molecule 1 (CEACAM1) in breast epithelial cells generating an immunoreceptor tyrosine-based inhibition motif (ITIM) containing isoform. Mol Cancer. 2014;13:64.

27. Nedellec P, Dveksler GS, Daniels E, Turbide C, Chow B, Basile AA, Holmes KV, Beauchemin N. Bgp2, a new member of the carcinoembryonic antigenrelated gene family, encodes an alternative receptor for mouse hepatitis viruses. J Virol. 1994;68(7):4525-37.

28. Sapoznik S, Hammer O, Ortenberg R, Besser MJ, Ben-Moshe T, Schachter J, Markel G. Novel anti-melanoma immunotherapies: disarming tumor escape mechanisms. Clin Dev Immunol. 2012;2012:818214.

29. Barclay AN, Hatherley D. The counterbalance theory for evolution and function of paired receptors. Immunity. 2008:29(5):675-8.

30. van Beek EM, Cochrane F, Barclay AN, van den Berg TK: Signal regulatory proteins in the immune system. J Immunol 2005, 175(12):7781-7787.

31. Barclay AN, Van den Berg TK. The interaction between signal regulatory protein alpha (SIRPalpha) and CD47: structure, function, and therapeutic target. Annu Rev Immunol. 2014;32:25-50.

32. Zebhauser R, Kammerer R, Eisenried A, McLellan A, Moore T, Zimmermann W. Identification of a novel group of evolutionarily conserved members within the rapidly diverging murine Cea family. Genomics. 2005;86(5):566-80.

33. Khairnar V, Duhan V, Maney SK, Honke N, Shaabani N, Pandyra AA, Seifert M, Pozdeev V, Xu HC, Sharma P, et al. CEACAM1 induces B-cell survival and is essential for protective antiviral antibody production. Nat Commun. 2015;6:6217.

34. Kammerer R, Stober D, Singer BB, Obrink B, Reimann J. Carcinoembryonic antigen-related cell adhesion molecule 1 on murine dendritic cells is a potent regulator of T cell stimulation. J Immunol. 2001;166(11):6537-44.

35. Jiang L, Barclay AN. Identification of leucocyte surface protein interactions by high-throughput screening with multivalent reagents. Immunology. 2010;129(1):55-61.

36. Singer BB, Opp L, Heinrich A, Schreiber F, Binding-Liermann R, BerrocalAlmanza LC, Heyl KA, Muller MM, Weimann A, Zweigner J, et al. Soluble CEACAM8 interacts with CEACAM1 inhibiting TLR2-triggered immune responses. PLoS One. 2014;9(4):e94106.

37. Markel G, Achdout H, Katz G, Ling KL, Salio M, Gruda R, Gazit R, Mizrahi S, Hanna J, Gonen-Gross T, et al. Biological function of the soluble CEACAM1 protein and implications in TAP2-deficient patients. Eur J Immunol. 2004; 34(8):2138-48.

\section{Submit your next manuscript to BioMed Central and we will help you at every step:}

- We accept pre-submission inquiries

- Our selector tool helps you to find the most relevant journal

- We provide round the clock customer support

- Convenient online submission

- Thorough peer review

- Inclusion in PubMed and all major indexing services

- Maximum visibility for your research

Submit your manuscript at www.biomedcentral.com/submit 